\title{
The photochemistry of 1-alkenyl-substituted-1,2,3-benzotriazoles leading to formation of indole and fused indole derivatives
}

\author{
Nader Al-Jalal,* Nouria A. Al-Awadi, Maher R. Ibrahim, \\ and Mohamed H. Elnagdi \\ Chemistry Department, Kuwait University, Kuwait University, P.O. Box 5969, \\ Safat 13060, Kuwait. \\ E-mail: nader.aljalal@ku.edu.kw
}

\begin{abstract}
Under photolysis conditions involving irradiation with a $16 \mathrm{~W}$ low pressure mercury arc-lamp $(254 \mathrm{~nm})$ or sunlight, functionally substituted 1-vinylbenzotriazoles react efficiently to produce 2-acylindoles, 2-benzotriazol-1-yl-4-methylquinolin-3-ol, isatin, indolo[2,1-b]quinazoline-6,12dione (tryptanthrin) and dihydropyrazolo[4,3-b]indole.
\end{abstract}

Keywords: Photolysis, 1-vinylbenzotriazoles, 2-acylindoles, 3-quinolinol, isatin, tryptanthrin, pyrazolo[4,3-b]indole

\section{Introduction}

It is generally known that 3-substituted indoles can be readily generated via reactions of indoles with electrophilic reagents and that 2-substituted indoles are better produced by using Nenitzescu, Madelung and Gassman ring synthesis methodologies. ${ }^{1}$ Earlier, we described a protocol for the synthesis of 3-acylindoles, ${ }^{2}$ in which difficultly obtained C-2 substituted indoles could be prepared.

Previous studies have shown that thermolytic and/or photolytic reactions of 1-substituted benzotriazole derivatives take place with elimination of $\mathrm{N}_{2}$ followed by subsequent ring closure of the resulting biradical intermediates to form heterocyclic products (Figure 1). ${ }^{3-8}$ These efficient processes have been described by Katritzky and his coworkers. ${ }^{9}$ In a follow up to our earlier efforts in which syntheses of the benzotriazole derivatives 1a-c, 10 and 15 were developed, ${ }^{10 \mathrm{a}-\mathrm{d}}$ we gained have explored the photochemistry of these substances, postulating that the reactions would afford 2-substituted indoles in good yields. Below, we report the results of this investigations aimed at the synthesis of substances of 2-substituted indoles through photochemical reactions of benzotriazoles 1a-c, $\mathbf{1 0}$ and $\mathbf{1 5 .}$ 


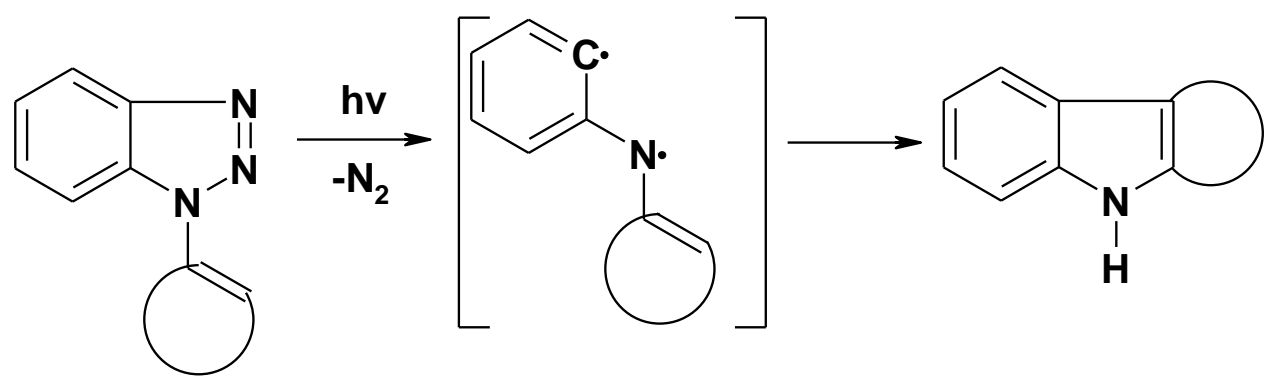

Figure 1

\section{Results and Discussion}

The 1-substituted benzotriazole derivatives 1a-c, $\mathbf{1 0}$ and 15, required in this study, were prepared following the procedures described by us earlier. ${ }^{10 a-d}$ These substances were fully characterized by using spectroscopic techniques. The UV spectra of these compounds display absorption maxima in the 248-296 $\mathrm{nm}$ wavelength regions (Table 1).

In Scheme 1 are summarized the results of reactions promoted by irradiation of acetonitrile solutions of 1a-c in quartz glass tubes with a $16 \mathrm{~W}$ low pressure mercury arc-lamp (254 nm) under a nitrogen atmosphere for $24 \mathrm{~h}$ at room temperature. Upon irradiation, benzotriazole 1a reacts to give 2-acetyl-3-dimethylaminoindole 3a (58\%) and 2-benzotriazolyl-4-methylquinolin3-ol 4 (15\%). The structure of 4 is well defined by its complete ${ }^{1} \mathrm{H}$ NMR, ${ }^{13} \mathrm{C}$ NMR, and mass spectrometric data, as well as by x-ray crystallographic analysis (Figure 4). In a similar manner, 1b and 1c undergo photochemical reactions to form only the 2-benzoyl- and 2-cyano-3dimethylaminoindole derivatives $\mathbf{3 b}$ and $\mathbf{3 c}$, respectively, in respective yields of $62 \%$ and $73 \%$ (Table 1). Products 3a-c can also be generated in 10-18\% yields by irradiation of acetonitrile solutions of 1a-c in Pyrex glass tubes using sunlight for 15 days (Table 1).

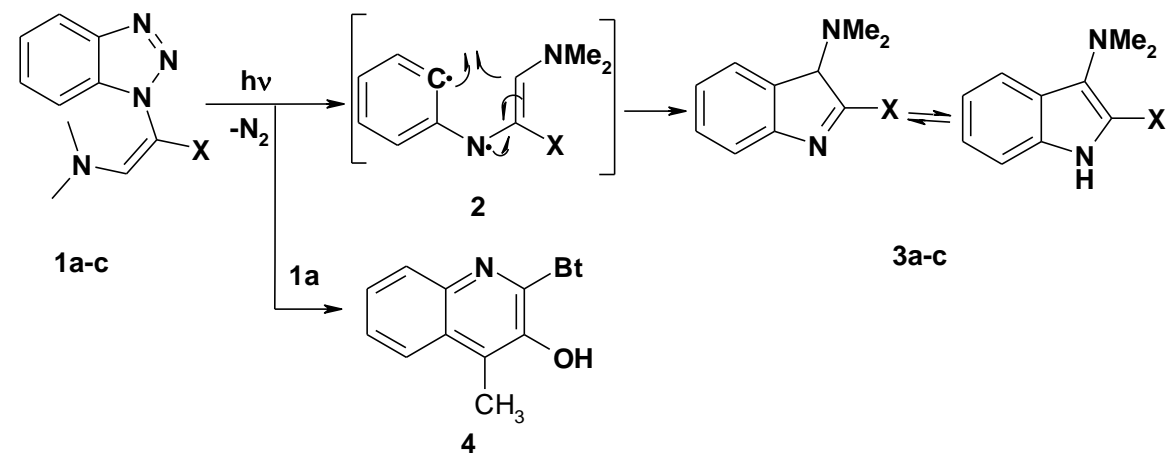

a, $\mathrm{X}=\mathrm{CH}_{3} \mathrm{CO} ; \mathrm{b}, \mathrm{X}=\mathrm{PhCO} ; \mathrm{c}, \mathrm{X}=\mathrm{CN} ; \mathrm{Bt}=1$-benzotriazolyl

Scheme 1. Photolysis of 1a-c. 
Table 1. Photoproducts formed by irradiation of $\mathbf{1 a - c ,} \mathbf{1 0}$ and $\mathbf{1 5}$

\begin{tabular}{lllll}
\hline Entry & Substrate & $\lambda_{\max }$ & Condition & $\begin{array}{l}\text { Product } \\
\text { (Yield) }\end{array}$ \\
\hline 1 & 1a & 286 & $\mathrm{~A}$ & $\mathbf{3 a}(58 \%)$ \\
& & & & $\mathbf{4}(15 \%)$ \\
2 & $\mathbf{1 a}$ & & $\mathrm{B}$ & $\mathbf{3 a}(18 \%)$ \\
3 & $\mathbf{1 b}$ & 296 & $\mathrm{~A}$ & $\mathbf{3 b}(62 \%)$ \\
4 & $\mathbf{1 b}$ & & $\mathrm{B}$ & $\mathbf{3 b}(10 \%)$ \\
5 & $\mathbf{1 c}$ & 278 & $\mathrm{~A}$ & $\mathbf{3 c}(73 \%)$ \\
6 & $\mathbf{1 c}$ & & $\mathrm{B}$ & $\mathbf{3 c}(16 \%)$ \\
7 & $\mathbf{1 0}$ & 274 & $\mathrm{~A}$ & $\mathbf{1 2}(41 \%)$ \\
& & & & $\mathbf{1 4}(38 \%)$ \\
8 & $\mathbf{1 5}$ & 248 & $\mathrm{~A}$ & $\mathbf{1 6}(68 \%)$ \\
\hline
\end{tabular}

A: Irradiation using a low pressure mercury arc-lamp $(254 \mathrm{~nm})$; B: Irradiation using sunlight

The structures of all new compounds were assigned by using spectroscopic and analytical methods. The structure of $\mathbf{3 a}$ is readily assigned based on $2 \mathrm{D}$ NMR results. The ${ }^{1} \mathrm{H}$ and ${ }^{13} \mathrm{C}$ signal assignments and the H-C correlations from the HMBC 2-D experiments are displayed in Figure 2. The important $\mathrm{HMBC}$ results include the observations that $\mathrm{H}-4$ at $7.83 \mathrm{ppm}$ correlates with C-2a, C-6 at 135.2 ppm, 126.1 ppm; H-7 at 7.25 ppm correlates with C-3a, C-5 at 124.3 ppm, 119.6 ppm; H-6 at 7.21 ppm correlates with C-2a, C-4 at 135.2 ppm, 122.9 ppm; H-5 at 6.99 ppm correlates with C-3a, C-7 at 124.3 ppm, 112.6 ppm; H-9 at 2.66 ppm correlates with C8, C-2 at 190.5 ppm, 128.7 ppm; H-10 at 2.98 ppm correlates with C-3 at 137.8 ppm. Finally, single crystal X-ray structure analysis (Figures 3 and 4) confirmed the structures of the new compounds $3 \mathrm{c}$ and $\mathbf{4}$.

\section{2-Acetyl-3-dimethylamino- $1 \mathrm{H}$-indole}
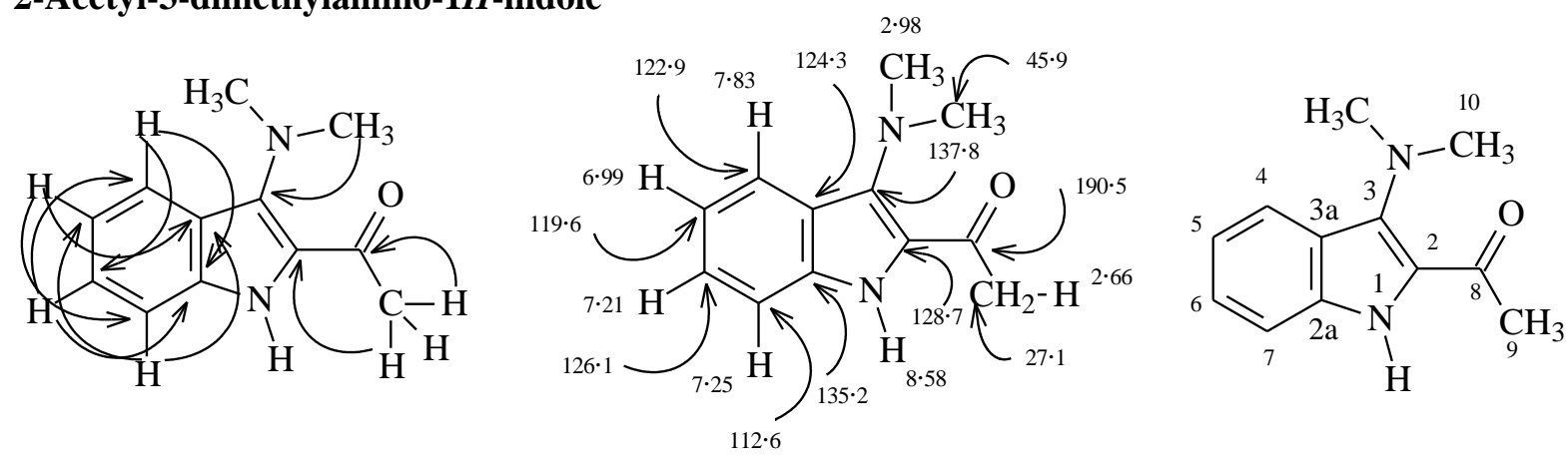

Figure 2. Important $\mathrm{HMBC}, \mathrm{H}-\mathrm{C}$ correlation of compound 3a. 


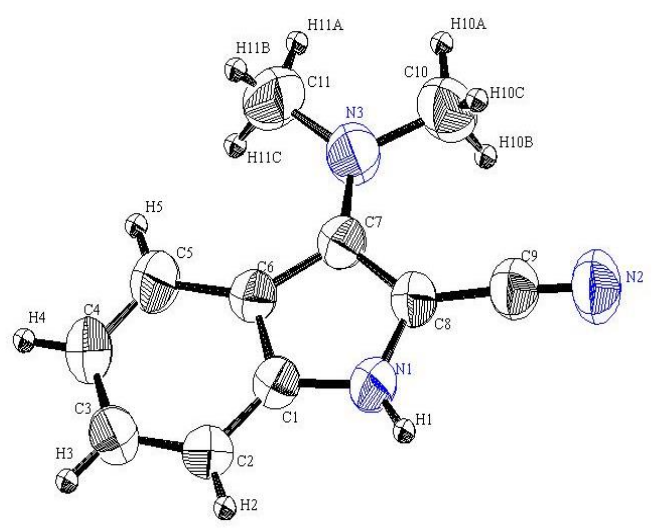

Figure 3. ORTEP drawing of 3c.

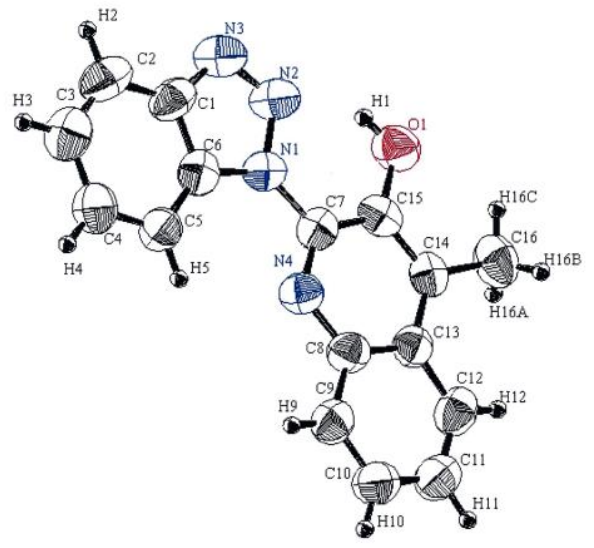

Figure 4. ORTEP drawing of 4.

The formation of 3a-c in these processes can be readily explained by a mechanism involving initial photo-extrusion of $\mathrm{N}_{2}$ to form the corresponding diradical intermediates 2 , which then cyclize followed by a $1,3-\mathrm{H}$ shift to yield the indole derivatives. On the other hand, formation of 2-benzotriazolyl-4-methylquinolin-3-ol 4 from 1a is likely a result of initial excited state N1-C bond cleavage to give radical 5 , which then reacts with another molecule of $1 \mathrm{a}$ to form $\mathbf{6}$. The latter intermediate then loses $\mathrm{N}_{2}$ and rearranges to give radicals 7, 8 and 9 sequentially and finally 4 (Scheme 2). 


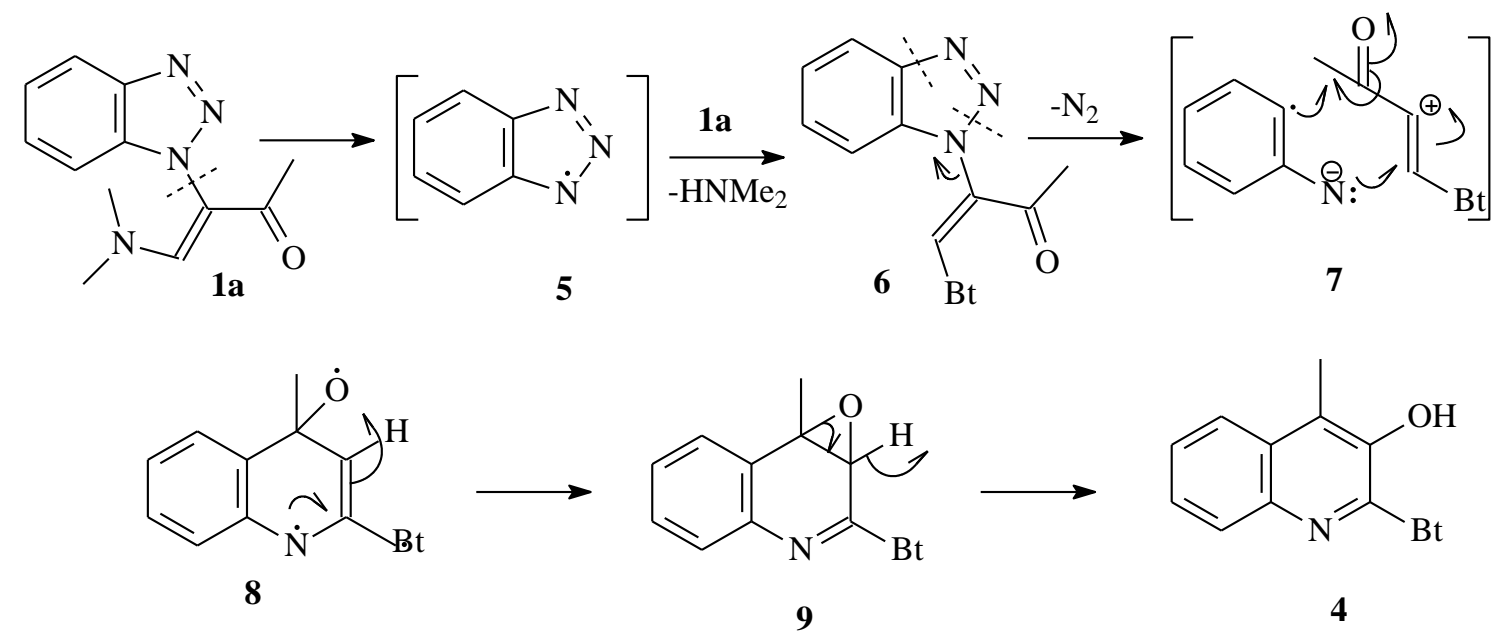

Scheme 2. Possible mechanism for the formation of 4.

Photochemical reaction of ethyl 2-benzotriazol-1-yl-3-dimethylaminoacrylate 10 gives isatin 12 (41\%) and indolo[2,1-b]quinazoline-6,12-dione (tryptanthrin) 14 (39\%) (Scheme 3, Table 1). A plausible route for formation of isatin involves the intermediacy of biradical 11. A similar pathway has been previously described. ${ }^{11}$ On the other hand, the tetracyclic product $\mathbf{1 4}$ is most likely formed by secondary photochemical reaction of $\mathbf{1 2}$ giving isatoic anhydride $\mathbf{1 3}$ that relies on the presence of traces of water in the reaction mixture. This assumption was probed by carrying out photoreaction of $\mathbf{1 2}$, which after a $24 \mathrm{~h}$ irradiation period gave $\mathbf{1 3}$ in quantitative yield. It has been previously reported that under basic conditions anhydride 13 reacts with 12 to produce 14. ${ }^{12 a}$ It should be noted that the present method serves as a direct route for the preparation of tryptanthrin $\mathbf{1 4}$, a well known and biologically interesting natural product. ${ }^{12 \mathrm{~b}}$

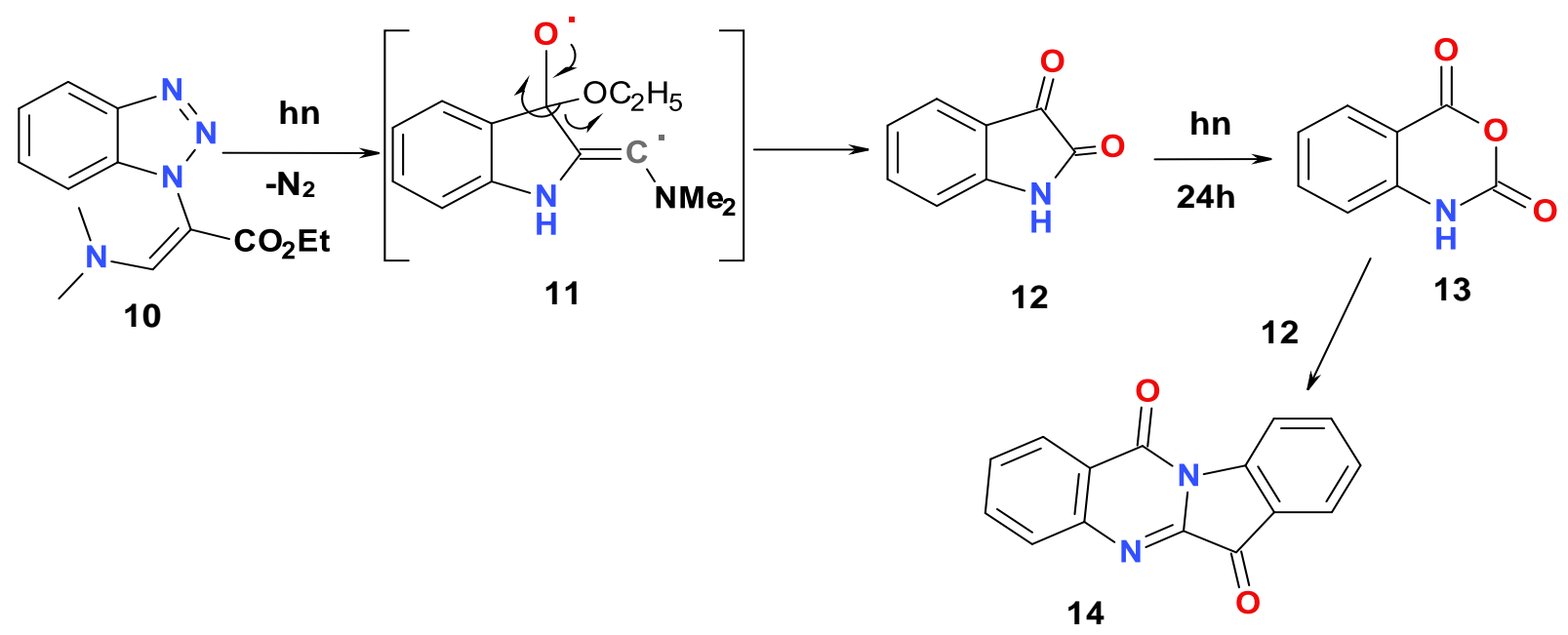

Scheme 3. Photolysis products of compound 10. 
The current studies were extended to include the exploration of the photochemistry of 1-(5phenyl-1H-pyrazol-4-yl)- $1 H$-benzotriazole 15, which was observed to undergo irradiation promoted $\mathrm{N}_{2}$ elimination to generate a biradical intermediate that cyclizes to yield the corresponding 3-phenyl-1,4-dihydropyrazolo[4,3-b]indole 16 in 68\% yield (Scheme 4).

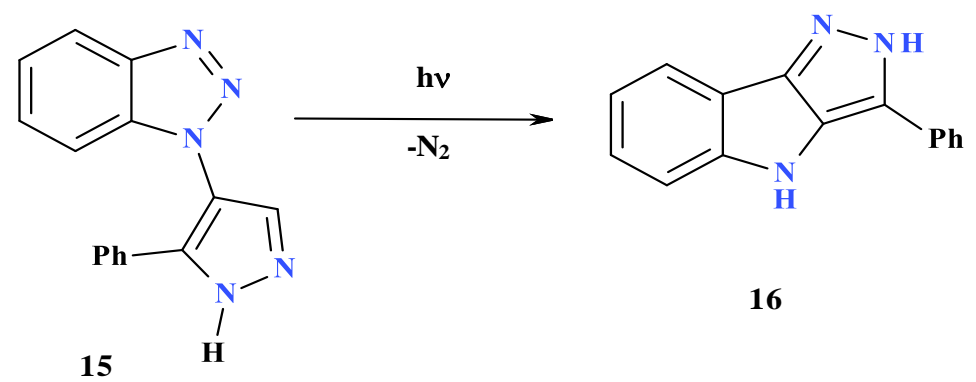

Scheme 4. Photolysis of 15.

\section{Conclusion}

The investigation described above has resulted in an efficient direct photochemical methodology for the preparation of new indole derivatives, some of which are difficult to obtain using other procedures. Also, a new interesting photochemical route for synthesis of the biologically active natural product tryptanthrin as well as other biologically and pharmaceutically interesting indole and condensed indole derivatives have been developed. ${ }^{13-15}$

\section{Experimental Section}

General. Melting points were recorded on a Gallenkamp apparatus. IR spectra were recorded using $\mathrm{KBr}$ pellets on a Perkin-Elmer $2000 \mathrm{FT}$-IR spectrophotometer. ${ }^{1} \mathrm{H}$ - and ${ }^{13} \mathrm{C}$ - NMR spectra were recorded on a Bruker DPX $400 \mathrm{MHz}$, Avance ${ }^{\mathrm{II}} 600 \mathrm{MHz}$ super-conducting NMR spectrometer with proton spectra measured at $400 \mathrm{MHz}$ and carbon spectra at 100 and $150 \mathrm{MHz}$. All chemical shifts are reported in ppm relative to tetramethylsilane (TMS) for ${ }^{1} \mathrm{H}$ or $\mathrm{CHCl}_{3}$ for ${ }^{13} \mathrm{C}$. IR data are reported in $\mathrm{cm}^{-1}$. Mass spectra were measured on a VG Auto-spec-Q (high resolution, high performance, tri-sector GC/MS/MS) and with LCMS using Agilent 1100 series LC/MSD with an API-ES/APCI ionization mode. Microanalysis were performed on a LECO CH NS-932 Elemental Analyzer. The UV/VIS absorption spectra were recorded using a Varian Cary 5 instrument. X-Ray analysis were performed using a Rigaku Rapid II diffractometer.

Starting materials. Starting compounds 1a-c, 10 and 15 were prepared using previously reported procedures. ${ }^{10 \mathrm{a}-\mathrm{d}}$ 
Preparation of (15). A mixture of $\mathbf{1 b}(2.92 \mathrm{~g}, 10 \mathrm{mmol})$ and hydrazine hydrate $(99 \%, 3 \mathrm{~mL})$ in ethanol $(25 \mathrm{~mL})$ was stirred at reflux for 3-4 h. Concentration of the mixture in vacuo gave a residue which was subjected to crystallization from ethanol.

1-(5-Phenyl-1H-pyrazol-4-yl)-1H-benzotriazole (15). Yield $2.0 \mathrm{~g}$ (76\%) from ethanol, $\mathrm{mp}$ 168-170 ${ }^{\circ} \mathrm{C}$. UV/VIS $\left(\mathrm{CHCl}_{3}\right): \lambda_{\max }=248 \mathrm{~nm} .{ }^{1} \mathrm{H}$ NMR (400 MHz, DMSO-d $)$ ): 13.58 (br, $1 \mathrm{H}$, $\mathrm{NH}), 8.36(\mathrm{~s}, 1 \mathrm{H}), 8.17(\mathrm{~d}, 1 \mathrm{H}, J 8.4 \mathrm{~Hz}), 7.53(\mathrm{t}, 1 \mathrm{H}, J 8.4 \mathrm{~Hz}), 7.47(\mathrm{t}, 1 \mathrm{H}, J 8.4 \mathrm{~Hz}), 7.40(\mathrm{~d}$, $1 \mathrm{H}, J 8.4 \mathrm{~Hz}), 7.27$ (m, 3H), $7.16(\mathrm{~m}, 2 \mathrm{H}) .{ }^{13} \mathrm{C}$ NMR (100 MHz, DMSO-d6): 145.3, 144.9, 134.3, 131.3, 129.2, 128.7, 128.5, 128.2, 126.1, 124.5, 119.5, 114.8, 110.4. MS: $m / z(\%) 261\left(\mathrm{M}^{+}, 40\right)$, 233 (100), 205 (50). Anal. Calc. for $\mathrm{C}_{15} \mathrm{H}_{11} \mathrm{~N}_{5}$ (261.3): C 68.95; H 4.24; $\mathrm{N}$ 26.80. Found: $\mathrm{C}$ $68.90 ; \mathrm{H} 4.19 ; \mathrm{N} 26.77 \%$.

\section{Photochemistry}

Method A. Irradiation using a low pressure mercury arc-lamp. Each of the substrates 1a-c, 10 and 15 (1.0 mmol) was dissolved in acetonitrile $(25 \mathrm{~mL})$ in a quartz tube and purged with nitrogen while being irradiated for $24 \mathrm{~h}$ at room temperature (RT). The progress of each reaction was monitored by using TLC. The solvent was removed in vacuo and the resulting residue was subjected to column chromatography on silica gel using ethyl acetate/ petroleum (b.p. $60-80{ }^{\circ} \mathrm{C}$ ) as the eluent to give the corresponding products (Table 1).

Method B. Irradiation using sunlight. Each of the substrates 1a-c $(0.5 \mathrm{~g})$ was dissolved in acetonitrile $(150 \mathrm{~mL})$ in a Pyrex tube, purged with nitrogen, and exposed to direct sunlight for 15 days in (July 1-15) at RT. The progress of each reaction was monitored by using TLC and LCMS. The solvent was removed in vacuo and the resulting residue was subjected to column chromatography on silica gel using ethyl acetate/ petroleum (b.p. $60-80{ }^{\circ} \mathrm{C}$ ) as eluent to give the products 3a-c in $10-18 \%$ yields (Table 1 ).

2-Acetyl-3-dimethylamino-1H-indole (3a). Yellow crystals from ethanol, mp $145-146{ }^{\circ} \mathrm{C}$, yield $58 \%(\operatorname{method} \mathrm{A}), 18 \%(\operatorname{method} \mathrm{B}),\left(\mathrm{R}_{\mathrm{f}} 0.48\right.$, EtOAc: petroleum b.p. $\left.40-60{ }^{\circ} \mathrm{C}: 1: 8 \mathrm{v} / \mathrm{v}\right)$. IR: 3337 , 3064, 2974, 1639, 1571, 1527, 1452, 1332, 1249, 1192, 975, 927, 744, 712. ${ }^{1} \mathrm{H}$ NMR (400 MHz, $\left.\mathrm{CDCl}_{3}\right): 8.58(\mathrm{br}, 1 \mathrm{H}, \mathrm{NH}), 7.83(\mathrm{~d}, 1 \mathrm{H}, J 8.0 \mathrm{~Hz}), 7.25(\mathrm{~d}, 1 \mathrm{H}, J 8.0 \mathrm{~Hz}), 7.21(\mathrm{t}, 1 \mathrm{H}, J 7.8 \mathrm{~Hz})$, $6.99(\mathrm{t}, 1 \mathrm{H}, J 7.8 \mathrm{~Hz}), 2.98\left(\mathrm{~s}, 6 \mathrm{H}, 2 \mathrm{CH}_{3}\right), 2.66\left(\mathrm{~s}, 3 \mathrm{H}, \mathrm{CH}_{3}\right) .{ }^{13} \mathrm{C} \mathrm{NMR}\left(100 \mathrm{MHz}, \mathrm{CDCl}_{3}\right)$ : 190.5, 137.8, 135.2, 128.7, 126.1, 124.3, 122.9, 119.6, 112.6, 45.9 (2C), 27.1. LCMS: $\mathrm{m} / z=203$ $(\mathrm{M}+1)$. MS: $m / z(\%) 202\left(\mathrm{M}^{+}, 100\right), 158$ (75), 105 (100). Anal. Calc. for $\mathrm{C}_{12} \mathrm{H}_{14} \mathrm{~N}_{2} \mathrm{O}$ (202.3): C 71.26; H 6.98; N 13.85. Found: C 71.20; H 6.90; N 13.79\%.

2-Benzotriazol-1-yl-4-methylquinolin-3-ol (4). Yield $15 \%$, (method A). Yellow crystals from ethanol: mp 174-176 ${ }^{\circ} \mathrm{C}$. ( $\mathrm{R}_{\mathrm{f}}$ 0.68, EtOAc: petroleum b.p. $\left.40-60{ }^{\circ} \mathrm{C}, 1: 5\right)$. IR: 3181, 3065, 2955 , 2860, 1728, 1451, 1379, 1272, 1122, 1072, 747 $\mathrm{cm}^{-1} .{ }^{1} \mathrm{H}$ NMR (600 MHz, $\left.\mathrm{CDCl}_{3}\right): 10.69$ (s, 1H, $\mathrm{OH}), 9.13(\mathrm{~d}, 1 \mathrm{H}, J 8.4 \mathrm{~Hz}), 8.25(\mathrm{~d}, 1 \mathrm{H}, J 8.4 \mathrm{~Hz}), 8.12(\mathrm{dd}, 1 \mathrm{H}, J 8.0,1,4 \mathrm{~Hz}), 8.00(\mathrm{dd}, 1 \mathrm{H}, J$ 8.0, $1.4 \mathrm{~Hz}), 7.79$ (t, $1 \mathrm{H}, J 8.0 \mathrm{~Hz}), 7.68-7.59(\mathrm{~m}, 3 \mathrm{H}), 2.78\left(\mathrm{~s}, 3 \mathrm{H}, \mathrm{CH}_{3}\right) .{ }^{13} \mathrm{C} \mathrm{NMR}(150 \mathrm{MHz}$, $\left.\mathrm{CDCl}_{3}\right): 145.1,140.6,140.5,139.0,132.0,130.4,129.8,128.9,128.4,127.2,126.9,126.1$, 123.2, 120.1, 116.4, 11.0. LCMS: $m / z=277(\mathrm{M}+1) . \mathrm{MS}: m / z(\%) 276\left(\mathrm{M}^{+}, 20\right), 248(45), 219$ 
(100). Anal. Calc. for $\mathrm{C}_{16} \mathrm{H}_{12} \mathrm{~N}_{4} \mathrm{O}$ (276.3): C 69.55; H 4.38; $\mathrm{N}$ 20.28. Found: C 69.49; H 4.34; $\mathrm{N}$ $20.27 \%$. HRMS = 276.1005, requires $\mathrm{C}_{16} \mathrm{H}_{12} \mathrm{~N}_{4} \mathrm{O} 276.1005$.

2-Benzoyl-3-dimethylamino-1H-indole (3b). Yellow crystals from ethanol: $\mathrm{mp}$ 197-198 ${ }^{\circ} \mathrm{C}$, yield $62 \%(\operatorname{method} A), 10 \%$ (method B), $\left(\mathrm{R}_{\mathrm{f}}\right.$ 0.47, EtOAc: petroleum b.p. $\left.40-60{ }^{\circ} \mathrm{C}: 3: 7\right)$. IR: 3337, 3064, 2974, 1639, 1571, 1527, 1452, 1332, 1249, 1192, 975, 927, 744, $712 \mathrm{~cm}^{-1}$. ${ }^{1} \mathrm{H}$ NMR $\left(400 \mathrm{MHz}, \mathrm{CDCl}_{3}\right): 8.08$ (d, 1H, J 8.4 Hz), 7.68 (d, 1H, J 8.0 Hz), 7.55 (d, 2H, J 8.0 Hz), 7.40$7.33(\mathrm{~m}, 3 \mathrm{H}), 7.26(\mathrm{t}, 2 \mathrm{H}, J 7.8 \mathrm{~Hz}), 7.03(\mathrm{t}, 1 \mathrm{H}, J 8.0 \mathrm{~Hz}), 3.28\left(\mathrm{~s}, 6 \mathrm{H}, 2 \mathrm{CH}_{3}\right) .{ }^{13} \mathrm{C} \mathrm{NMR}(100$ $\left.\mathrm{MHz}_{\mathrm{CDCl}}\right)$ : 190.6, 151.7, 138.5, 134.4, 130.0, 128.1, 127.9, 127.7, 127.3, 125.9, 123.6, 119.2, 108.4, 43.0 (2C). LCMS: $m / z=265(\mathrm{M}+1)$. MS: $m / z(\%) 264\left(\mathrm{M}^{+}, 20\right), 208$ (65), 105 (50), 77 (100). (HRMS = 264.1258, requires $\left.\mathrm{C}_{17} \mathrm{H}_{16} \mathrm{~N}_{2} \mathrm{O} 264.1257\right)$.

3-Dimethylamino-1H-indole 2-carbonitrile (3c). Colorless crystals, mp 91-92 ${ }^{\circ} \mathrm{C}$, yield $73 \%$ (method A), $16 \%$ (method B), ( $\mathrm{R}_{\mathrm{f}}$ 0.57, EtOAc: petroleum bp 40-60 $\left.{ }^{\circ} \mathrm{C}: 1: 9\right) . \mathrm{IR}: 3305,3067$, 2924, 2202, 1571, 1549, 1455, 1339, 1219, 1137, 915, 744. ${ }^{1} \mathrm{H}$ NMR (600 MHz, CDCl $): 7.73$ (d, 1H, J $8.4 \mathrm{~Hz}), 7.71(\mathrm{br}, 1 \mathrm{H}, \mathrm{NH}), 7.33(\mathrm{dt}, 1 \mathrm{H}, J$ 8.4, $1.2 \mathrm{~Hz}), 7.26(\mathrm{~d}, 1 \mathrm{H}, J 8.4 \mathrm{~Hz}), 7.10$ (dt, $1 \mathrm{H}, J$ 8.0, $1.2 \mathrm{~Hz}), 3.18$ (s, 6H, 2CH$).{ }^{13} \mathrm{C} \mathrm{NMR}\left(100 \mathrm{MHz}, \mathrm{CDCl}_{3}\right)$ : 142.6, 137.2, 126.6, 121.6, 120.7, 120.0, 116.8, 111.9, 91.9, 43.8 (2C). MS: m/z (\%) $185\left(\mathrm{M}^{+}, 100\right), 170$ (60), 142 (50). Anal. Calc. for $\mathrm{C}_{11} \mathrm{H}_{11} \mathrm{~N}_{3}$ (185.2): C 71.33; H 5.99; N 22.69. Found: C 71.23; H 5.90; N 22.63\%. (HRMS = 185.0948, requires $\mathrm{C}_{11} \mathrm{H}_{11} \mathrm{~N}_{3}$ 185.0947).

$\mathbf{1 H}$-Indol-2,3-dione (Isatin) (12). Red brown crystals, mp 196-197 ${ }^{\circ} \mathrm{C}$ (lit. ${ }^{16} 195-197{ }^{\circ} \mathrm{C}$ ). Isatoic anhydride (13). Colorless crystals from ethanol, mp $243-45^{\circ} \mathrm{C}$ (lit. ${ }^{12 \mathrm{a}} 243-47{ }^{\circ} \mathrm{C}$ ).

Indolo[2,1-b]quinazoline-6,12-dione (Tryptanthrin) (14). Greenish yellow needles, mp 265$267{ }^{\circ} \mathrm{C}$ (lit. ${ }^{12 \mathrm{~b}} \mathrm{mp} \mathrm{266-267}{ }^{\circ} \mathrm{C}$ ). IR: 3020, 2938, 1732, 1691, 1585, 1462, 1314, 1194, 1110, 1036, 916, 751. ${ }^{1} \mathrm{H}$ NMR (600 MHz, $\left.\mathrm{CDCl}_{3}\right): 8.65$ (d, 1H, J 8.0 Hz), 8.47 (dd, 1H, J 8.0, $\left.1.2 \mathrm{~Hz}\right), 8.06$ $(\mathrm{dd}, 1 \mathrm{H}, J 8.0,1.2 \mathrm{~Hz}), 7.94(\mathrm{dd}, 1 \mathrm{H}, J 7.8,1.0 \mathrm{~Hz}), 7.88(\mathrm{dt}, 1 \mathrm{H}, J 7.8,1.2 \mathrm{~Hz}), 7.80(\mathrm{dt}, 1 \mathrm{H}, J$ 7.8, $1.2 \mathrm{~Hz}), 7.71(\mathrm{t}, 1 \mathrm{H}, J 8.0 \mathrm{~Hz}), 7.46(\mathrm{t}, 1 \mathrm{H}, J 7.8 \mathrm{~Hz}) .{ }^{13} \mathrm{C} \mathrm{NMR}\left(150 \mathrm{MHz}, \mathrm{CDCl}_{3}\right): 182.6$, 158.1, 146.7, 146.4, 144.4, 138.3, 135.1, 130.8, 130.3, 127.6, 127.2, 125.4, 123.8, 121.9, 118.0. MS: $m / z(\%) 248\left(\mathrm{M}^{+}, 100\right), 220$ (30), 192 (15). Anal. Calc. for $\mathrm{C}_{15} \mathrm{H}_{8} \mathrm{~N}_{2} \mathrm{O}_{2}$ (248.28): C 72.58; $\mathrm{H}$ 3.25; N 11.28. Found: C 72.50; H 3.24; N 11.19\%.

3-phenyl-1,4-dihydropyrazolo[4,3-b]indole (16). Colorless crystal from ethanol, mp 195-197 ${ }^{\circ} \mathrm{C}$, yield 68\% (method A), ( $\mathrm{R}_{\mathrm{f}}$ 0.47, EtOAc: petroleum, bp 40-60: 1 : 5). IR: 3020, 2938, 1732, 1691, 1595, 1462, 1314, 1194, 1110, 1036, 916, 751. ' H NMR (400 MHz, DMSO-d6): 12.98 (br, 1H, NH), 10.97 (br, 1H, NH), 7.98 (d, 2H, J 7.8 Hz), 7.77 (d, 1H, J 7.8 Hz), 7.50 (d, 1H, J 7.8 $\mathrm{Hz}), 7.46(\mathrm{t}, 2 \mathrm{H}, J 7.8 \mathrm{~Hz}), 7.29(\mathrm{~m}, 2 \mathrm{H}), 7.09(\mathrm{t}, 1 \mathrm{H}, J 7.8 \mathrm{~Hz}) .{ }^{13} \mathrm{C} \mathrm{NMR}\left(100 \mathrm{MHz}, \mathrm{CDCl}_{3}\right)$ : 145.3, 131.7, 130.9, 129.1, 128.7, 127.9, 127.7, 125.5, 125.4, 119.9, 119.7, 114.7, 112.5. MS: $m / z(\%) 233\left(\mathrm{M}^{+} 100\right), 205$ (10), 103 (25). Anal. Calc. for $\mathrm{C}_{15} \mathrm{H}_{11} \mathrm{~N}_{3}$ (233.3): C 77.23; $\mathrm{H} 4.75 ; \mathrm{N}$ 18.01. Found: C 77.20; H 4.68; N 18.07. 


\section{Acknowledgements}

Support from the University of Kuwait, received through Research Grant no. SC 04/08, and the facilities of ANALAB/SAF (grants no. GS01/01, GS02/01, GS03/08) are gratefully acknowledged

\section{References and Notes}

1. (a) Li, J. J. Name Reactions, 4th. ed., Springer, Berlin 2009, 391. (b) Seijas, J. A.; VazquezTato, M. P.; Crecente-Campo, J.; Gomez-Doval, M. G.; Nunez-Alvarez, L. $12^{\text {th }}$ International Electronic Conference on synthetic organic Chemistry (ECSOC-12) 1-30 November 2008, and references cited. (c) Li, J. J. Name Reactions. 4th. ed., Springer, Berlin 2009, page 251.

2. Abdel-Motaleb, R. M.; Makhloof, A. A.; Ibrahim, H. M.; Elnagdi, M. H. J. Heterocyclic Chem. 2007, 44, 109.

3. Dib, H. H.; Al-Awadi, N. A.; Ibrahim, Y. A.; El-Dusouqui, O. M. Tetrahedron 2003, 59, 9455.

4. Dib, H. H.; Al-Awadi, N. A.; Ibrahim, Y. A.; El-Dusouqui, O. M. J. Phys. Org. Chem. 2004, 17, 267.

5. Al-Awadi, N. A.; George, B.; Dib, H. H.; Ibrahim, M. R.; Ibrahim, Y. A.; El-Dusouqui, O. M. Tetrahedron 2005, 61, 8257.

6. Al-Awadi H.; Ibrahim, M. R.; Ibrahim, Y. A.; Al-Awadi, N. A. J. Heterocyclic Chem. 2008, $45,727$.

7. (a) Wender, P. A.; Cooper, C. B. Tetrahedron 1986, 42, 2985. (b) Märky, M.; Schmid, H.; Hansen, H. J. Helv. Chim. Acta 1979, 62, 2129.

8. Orlewska, C.; Saczewski, F. J. Heterocyclic Chem. 1993, 30, 833.

9. Katritzky, A. R.; Lan, X.; Yang, J.; Denisko, O. Chem. Rev. 1998, 98, 409.

10. (a) Al-Saleh, B.; Behbehani, H.; El-Apasery, M.; Elnagdi, M. H. J. Chem. Res. 2004, 575.

(b) Hassanien, A. Z.; Ghozlan, S. A.; Elnagdi, M. H. J. Chin. Chem. Soc. 2004, 51, 575. (c) Al-Omran, F.; Abd El-Hay, O. Y.; El-Khair, A. J. Heterocyclic Chem. 2000, 37, 167. (d) Gompper, R.; Walther, P.; Brauchle, C.; Stadler, S. Tetrahedron 1996, 52, 14607.

11. (a) Wilson, R. M.; Heng, A. C. J. Org. Chem. 1990, 55, 197. (b) Hewawasam, P.; Meanwell, N. A. Tetrahedron Lett. 1994, 35, 7303.

12. (a) Kumar, A.; Tripathi, V. D.; Kumar, P. Green Chemistry, 2011, 13, 51. (b) Potewar, M. T.; Ingale, A. S.; Srinivasan, V. K. Arkivoc, 2008, xiv, 100.

13. Horton, D. A.; Bourne, G. T.; Smythe, M. L. Chem. Rev. 2003, 103, 893.

14. Patel, A.; Bari, S.; Talele, G.; Patel, J.; Sarangapani, M. Iran J. Pharm. Res. 2006, 4, 249.

15. Doyle, K. J.; Rafferty, P.; Steele, R. W.; Wilkins, D. J.; Hockley, M.; Arnold, L. D.; Ericsson A. M. PCT int. Appl. 2000, 210. Chem. Abstr. 2000, 132, 347566.

16. Gassman, P. G.; Halweg, K. M. J. Org. Chem. 1979, 44, 628. 
17. Crystallographic data of for the structures (excluding structure factors) in this paper have been deposited with the Cambridge Crystallographic Data Centre as supplementary publication numbers CCDC 836134 3c and CCDC 836135 4. Copies of the data can be obtained, free of charge, on application to CCDC, 12 Union Road, Cambridge CB2 1EZ, UK, (fax: +44-(0)1223-336033 or e-mail: deposit@ccdc.cam.ac.UK). 